\title{
Influence de l'élagage sur la duraminisation, la production de bois de tension et quelques autres propriétés du bois de peuplier 1214
}

\author{
H. POLGE \\ avec la collaboration technique de Thérèse HURPEAU \\ I.N.R.A., Station de Recherches sur la Qualité des Bois \\ Centre de Recherches forestières, Champenoux, F 54280 Seichamps
}

\begin{abstract}
Résumé
Quatre carottes de sondage ont été prélevées (à $1,30 \mathrm{~m}$ et $6,50 \mathrm{~m}$ de hauteur à la fois sur le côté Est et sur le côté Ouest des tiges) dans 177 peupliers I 214 soumis à différents taux d'élagage pour connaître les effets de ce traitement, plus particulièrement sur le développement du bois de tension (estimé indirectement à partir du diamètre tangentiel des carottes à l'état frais) et sur la formation du bois de cour; les principaux résultats sont les suivants :

- le bois de coeur est plus large et l'aubier plus mince; le retrait et la densité sont plus élevés sur le côté Ouest des arbres, c'est-à-dire celui d'où viennent les vents dominants ; d'homme ;

- à $6,50 \mathrm{~m}$, les cernes d'aubier sont moins nombreux, mais plus larges qu'à hauteur

- l'élagage influence les divers paramètres se rapportant à la formation du bois de couur; dans les arbres élagués à 65 p. 100, l'aubier s'étend sur un plus grand nombre d'accroissements, mais ceux-ci sont plus étroits que dans les autres traitements;

- une tendance apparaît pour que les arbres sévèrement élagués (aux taux de 55 et 65 p. 100) aient un pourcentage plus élevé de bois de cœur;

- le bois de tension est plus abondant sur le côté Ouest des tiges en relation avec les contraintes de tension dues aux vents, mais son développement est tout à fait indépendant du traitement d'élagage.
\end{abstract}

\section{Introduction}

La Station de Recherches sur la Qualité des Bois a implanté en Forêt Domaniale de Retz deux dispositifs expérimentaux d'élagage de branches vivantes sur des peupliers $\mathrm{I} 214$ alors âgés de 7 ans. Une première analyse des résultats a été entreprise 7 ou 8 ans après le début des traitements (KELLER, 1979); elle fournissait de nombreux renseignements d'ordre dendrométrique, auxquels le lecteur pourra se reporter, permettant de classer le peuplement en cause parmi les peupleraies de belle venue, même s'il se révèle, au point de vue performances de croissance, quelque 
peu inférieur aux maxima obtenus notamment sur des sols mieux alimentés en eau; concernant les effets de l'élagage, elle montrait surtout :

- que l'élagage procure une diminution de la décroissance métrique d'autant plus marquée que son taux est plus élevé et que le défilement initial était important,

- qu'il n'y a aucun effet du traitement sur la croissance en hauteur,

- que le ralentissement de la croissance en circonférence au voisinage de la base de la cime vivante n'est sensible que lorsque l'ablation des branches vivantes porte sur 60 ou 65 p. 100 de la hauteur totale,

- que les effets de cette opération sur les variations de la densité du bois à l'intérieur des cernes sont à la fois peu marqués et peu durables,

- que, conséquence des observations précédentes, il est possible de préconiser des taux d'élagage sensiblement plus sévères que ceux précédemment donnés dans la littérature (PourTet, 1961).

Deux publications étrangères avaient cependant fait état d'une influence possible de l'élagage sur la duraminisation d'une part, et la production de bois de tension d'autre part, dans différents clones de peuplier :

Une première comparaison ne portant malheureusement que sur un arbre élagué et un témoin par cultivar avait donné les résultats suivants (SACHSSE, 1965) :

\begin{tabular}{l|c|c|c|c|c|c}
\hline \hline \multirow{2}{*}{$\%$ bois de cour } & Cultivar & Régénéré & Robusta & Oxford & Rochester & Wettstein \\
\cline { 2 - 5 } & Elagué ..... & 33,3 & 28,1 & 23,2 & 13,9 & 12,8 \\
& Non élagué . & 30,6 & 25,1 & 18,5 & 5,8 & 5,9 \\
& Elagué ...... & 10,9 & 9,6 & 15,4 & 15,4 & 33,9 \\
& Non élagué . & 3,0 & 8,7 & 4,0 & 13,0 & 11,1 \\
\hline
\end{tabular}

Les différences trouvées entre traitements étaient significatives pour les deux critères en cause ; l'accélération de la duraminisation liée à l'élagage était particulièrement sensible pour les trois cultivars hybrides de peupliers noirs et de baumiers où le pourcentage initial de bois de cour était le plus faible; quant au bois de tension, il apparaissait comme ayant triplé de surface à la suite du traitement d'élagage pour trois des variétés étudiées, ce qui est un résultat évidemment très inquiétant.

La deuxième étude (SACRE, 1974) ne concernait que le seul peuplier Robusta, et portait sur 5 arbres élagués et 5 témoins, malheureusement soumis à des conditions de compétition et de milieu très différentes : plantation en fond de vallée à $10 \times 10 \mathrm{~m}$ pour les premiers, et sur plateau à $4 \times 5 \mathrm{~m}$ pour les seconds. Les arbres élagués avaient 27 p. 100 de bois de coeur et 51 p. 100 de bois de tension, contre respectivement 19 p. 100 et 36 p. 100 pour les témoins.

Les deux séries d'observations allant dans le même sens défavorable à l'élagage, surtout pour le bois de tension qui engendre des déformations lors de la libération des contraintes de croissance et de séchage et un mauvais état de surface 
lors du sciage ou du rabotage (bois «pelucheux»), il a paru utile de chercher à savoir si des phénomènes analogues se produisaient sur le clone I 214, dans les conditions de culture de la Forêt de Retz.

\section{Matériel et méthodes}

L'étude a été entreprise au printemps 1983 dans le dispositif en lignes déjà décrit par ailleurs (Keller, op. cit.), dont il suffit de rappeler qu'il comporte une séquence de traitements : témoin, arbres élagués à $35,45,55$ et 65 p. 100 de la hauteur totale, répétée 38 fois, avec un premier élagage pratiqué au printemps 1969; à l'âge de 7 ans, réactualisé deux fois, aux mêmes taux, aux printemps 1974 et 1977 . Par suite de malformations ou de disparition de certains individus, l'échantillonnage s'est trouvé limité à un total de 177 individus (respectivement 36, 34, 34, 38 et 35 pour les 5 modalités de traitement indiquées ci-dessus), et la plupart des analyses statistiques n'ont porté que sur 170 arbres $(5 \times 34)$, de manière à obtenir un dispositif orthogonal, en éliminant, dans chaque catégorie de traitement où l'on disposait d'excédents, les derniers individus dans l'ordre des numéros, pour ne pas biaiser les résultats.

A cause de l'importance de l'échantillonnage, il ne pouvait être question d'abattre les arbres d'expérience, et les différentes analyses ont été faites sur carottes de sondage de $5 \mathrm{~mm}$ de diamètre ; compte tenu du fait que l'un des facteurs que l'on souhaitait étudier, le bois de tension, est lié à l'inclinaison des tiges, elle-même fonction des vents dominants, les prélèvements ont porté à la fois sur le côté Ouest (côté tendu) et Est (côté comprimé); ils ont été réalisćs à deux niveaux : 1,30 et $6,50 \mathrm{~m}$, donnant un total de 708 carottes.

\subsection{Données concernant la duraminisation}

La délimitation de la partie duraminisée des carottes a été faite aussitôt après prélèvement sur la base du changement de couleur qui la caractérise; la largeur de l'aubier a ensuite été mesurée au laboratoire, puis le nombre d'années correspondant, pour la détermination duquel le recours à la radiographie a paru nécessaire en raison des difficultés de reconnaissance des limites de cernes chez le peuplier.

\subsection{Estimation indirecte du bois de tension}

Le nombre de prélèvements à étudier était tel que la mesure directe du pourcentage de bois de tension, qui, sur carotte de sondage, ne peut se faire qu'après réalisation de coupes microscopiques, n'a pas semblé possible; on a donc utilisé, comme estimateur du bois de tension, le diamètre tangentiel à l'état frais des carottes de sondage, mesuré avec une précision du micron sur 100 points également répartis entre 5 et $25 \mathrm{~mm}$ depuis l'écorce (Perrin \& Ferrand, 1984). De premières recherches sur le hêtre avaient en effet fait apparaître des liaisons très étroites entre le pourcentage de bois de tension et les microdéformations liées à la libération des contraintes de croissance longitudinales (TrEnARD \& GUENEAU, 1975), puis entre 
celles-ci et le diamètre tangentiel à l'état frais de carottes de sondage prélevées au même niveau (Polge \& Thiercelin, 1979). Cet ensemble de résultats s'est trouvé confirmé, précisément pour le peuplier, par FERRAND (1981), qui a établi les corrélations suivantes :

- entre diamètre tangentiel et microdéformations : $\mathrm{r}=-0,77^{* * *}$,

- entre diamètre tangentiel et pourcentage de bois de tension $: \mathrm{r}=-0,76^{* * *}$,

- entre pourcentage de bois de tension et microdéformations : $r=0,77 * * *$.

Sur cette même espèce, OKuyama (1983) a trouvé entre le diamètre tangentiel frais et les microdéformations longitudinales des coefficients de corrélation allant de $-0,76^{* * *}$ à $-0,92 * * *$.

Enfin, dans un travail en cours à la Station de Recherches sur la Qualité des Bois, portant sur 34 peupliers de divers clones, une liaison très étroite a été mise en évidence entre le diamètre tangentiel et l'importance du bois pelucheux, qui est synonyme de bois de tension $\left(\mathrm{r}=-0,81^{* * * *}\right)$.

Ces corrélations, même si elles sont toutes très significatives, ne sont certes pas suffisantes pour permettre une appréciation indirecte du bois de tension au niveau individuel, mais elles sont assurément assez fortes pour justifier l'utilisation du diamètre tangentiel comme critère de comparaisons entre des groupes d'arbres comprenant au moins 34 individus chacun.

La mesure des diamètres tangentiels, avec l'extrême précision qui est requise pour ce genre d'analyses, exige évidemment une grande rigueur expérimentale. Au cas particulier, it n'y avait aucun problème pour l'ensemble des prélèvements à hauteur d'homme, et pour ceux effectués à $6,50 \mathrm{~m}$ sur les plus gros arbres, qui ont été réalisés séparément pour les côtés Est et Ouest. En revanche, on a procédé à des sondages diamétraux chaque fois que cela a été possible au niveau supérieur des plus petits individus de manière à économiser temps et fatigue des opérateurs qui n'avaient plus à positionner deux fois les échelles de grimpage pour un même arbre. Dans ce cas, on a pensé qu'il y avait un risque de biais systématique entre le côté entrée et le côté sortie de la tarière par suite de la dilatation du métal sous l'effet du frottement prolongé dans le bois notamment. A titre de précaution, on a cherché à équilibrer les entrées et les sorties Est et Ouest, et on a abouti, pour les prélèvements à $6,50 \mathrm{~m}$, à la répartition suivante :

— côté Est : 124 carottes «entrée» et 53 «sortie»,

一 côté Ouest : 125 carottes «entrée» et 52 «sortie».

\subsection{Autres mesures}

Les retraits axiaux et tangentiels ont été calculés à partir des diamètres moyens mesurés entre 5 et $25 \mathrm{~mm}$ depuis l'écorce, d'abord à l'état frais, puis après conditionnement à 10 p. 100 d'humidité par séjour jusqu'à poids constant dans une étuve hygrométrique dont les températures sèche et humide étaient réglées à $50^{\circ}$ et $42^{\circ}$ respectivement. Le retrait radial a été obtenu de la même façon sur la longueur de carotte représentée par l'aubier. 
L'infra-densité a été calculée sur la base du poids anhydre $P_{0}$ et poids maximum $\mathrm{P}_{\mathrm{II}}$ trouvé à l'issue d'une saturation par le vide de 96 heures par la formule :

$$
\text { Infra-densité }=\frac{1}{\frac{P_{\mathrm{m}}}{\mathrm{P}_{\mathrm{o}}}-0,347}
$$

\section{Résultats}

Compte tenu d'un effet «tarière» possible au niveau supérieur de sondage pour certaines variables (diamètres longitudinaux et tangentiel) suivant que le côté correspondant est celui d'une entrée ou d'une sortie de l'instrument, les diverses variables étudiées ont fait l'objet d'analyses de variances différentes.

\subsection{Analyse de variance sur la totaltié de l'échantillonnage pour les variables à effet tarière exclu}

Elle permet d'analyser les effets des 3 sources de variation (côté, niveau et traitement) et des 3 interactions d'ordre 2 ; ses résultats sont résumés dans le tableau suivant qui donne les valeurs des tests $« F »$ correspondants :

Tableau 1

\begin{tabular}{|c|c|c|c|c|c|c|c|}
\hline \multirow{2}{*}{\multicolumn{2}{|c|}{ Caractéristique analysée }} & \multicolumn{3}{|c|}{ Effets principaux } & \multicolumn{3}{|c|}{ Interactions } \\
\hline & & Côté & Niveau & Traitement & N.C. & T.C. & T.N. \\
\hline \multicolumn{2}{|l|}{ Longueur radiale du bois de cœur } & $4,99 *$ & $674,5 * *$ & $8,51 * *$ & N.S. & N.S. & N.S. \\
\hline \multicolumn{2}{|l|}{ Epaisseur de l'aubier . . . . . . . . } & $8,45^{*} *$ & $13,1 * k$ & $21,08^{* *}$ & N.S. & N.S. & N.S. \\
\hline \multicolumn{2}{|l|}{ Nombre de cernes de l'aubier .... } & N.S. & $258,2 * *$ & $8,34 * *$ & N.S. & N.S. & N.S. \\
\hline \multicolumn{2}{|l|}{ Largeur des cernes dans l'aubier .. } & N.S. & $53,7 * *$ & $29,77^{* *}$ & $4,1 *$ & N.S. & N.S. \\
\hline \multicolumn{2}{|l|}{ Pourcentage de bois de coeur .... } & $9,56 * *$ & $238,6 * *$ & N.S. & $3,9 *$ & N.S. & N.S. \\
\hline \multicolumn{2}{|l|}{ Retrait tangentiel $\ldots \ldots \ldots \ldots$} & $10,97 * *$ & $90,1 * *$ & N.S. & N.S. & N.S. & N.S. \\
\hline \multicolumn{2}{|l|}{ Retrait longitudinal . . . . . . . } & N.S. & $59,2 * *$ & N.S. & $5,0^{*}$ & N.S. & N.S. \\
\hline \multicolumn{2}{|l|}{ Retrait radial $\ldots \ldots \ldots \ldots \ldots \ldots$} & N.S. & $8,8^{* *}$ & N.S. & N.S. & N.S. & N.S. \\
\hline \multicolumn{2}{|l|}{ Infra-densité $\ldots \ldots \ldots \ldots \ldots \ldots$} & $7,56 * *$ & $12,1 * *$ & N.S. & N.S. & N.S. & N.S. \\
\hline \multirow{15}{*}{\multicolumn{2}{|c|}{$\begin{array}{ll}\text { Caractéristique analysée } & = \\
\text { Longueur radiale du bois de coeur } \\
\text { Epaisseur de l'aubier } \\
\text { Nombre de cernes de l'aubier } \\
\text { Largeur de cernes dans l'aubier } \\
\text { Pourcentage de bois de coeur } \\
\text { Retrait tangentiel } \\
\text { Retrait longitudinal } \\
\text { Retrait radial } \\
\text { Infra-densité } \\
\text { Effets principaux } \\
\text { Interactions } \\
\text { Côté } \\
\text { Niveau } & = \\
\text { Traitement } & = \\
& =\end{array}$}} & property a & zed. & & & & \\
\hline & & & f hear & & & & \\
\hline & & sapwood th & kness. & & & & \\
\hline & & niumber of & pwood rin & & & & \\
\hline & & sapwood ri & width. & & & & \\
\hline & & percentage & heartwoo & & & & \\
\hline & & tangentials & inkage. & & & & \\
\hline & & longitudinal & hrinkage. & & & & \\
\hline & & radial shrin & ge. & & & & \\
\hline & & specific gra & & & & & \\
\hline & & main effect & & & & & \\
\hline & & interaction. & & & & & \\
\hline & & side. & & & & & \\
\hline & & height. & & & & & \\
\hline & & treatment. & & & & & \\
\hline
\end{tabular}


La diminution du bois de cour quand la hauteur dans l'arbre augmente est normale (il tend vers zéro dans la partie la plus jeune du tronc); la réduction du nombre de cernes d'aubier à $6,50 \mathrm{~m}$ apparaît comme un corollaire de leur plus grande largeur (elle-même liée à la plus grande proximité des sources de produits de la photosynthèse et des substances de croissance), si l'on admet l'hypothèse de BAmBER (1975) suivant laquelle la surface de l'aubier est limitée, par des phénomènes régulatoires, au strict minimum nécessaire pour assurer la circulation de la sève et l'approvisionnement des cellules en substances nutritives.

La réduction des retraits tangentiel et radial, de même que l'augmentation du retrait longitudinal et de la densité avec la hauteur correspondent à des effets de gradient normaux liés au caractère plus juvénile du bois présent aux niveaux supérieurs.

Quatre variables seulement sont soumises à un effet traitement significatif ; les tests de comparaison de moyennes les concernant donnent les résultats suivants (les pourcentages indiqués correspondent aux taux d'élagage, et les seuils de signification sont donnés en bout de ligne) :

TABLEAU 4

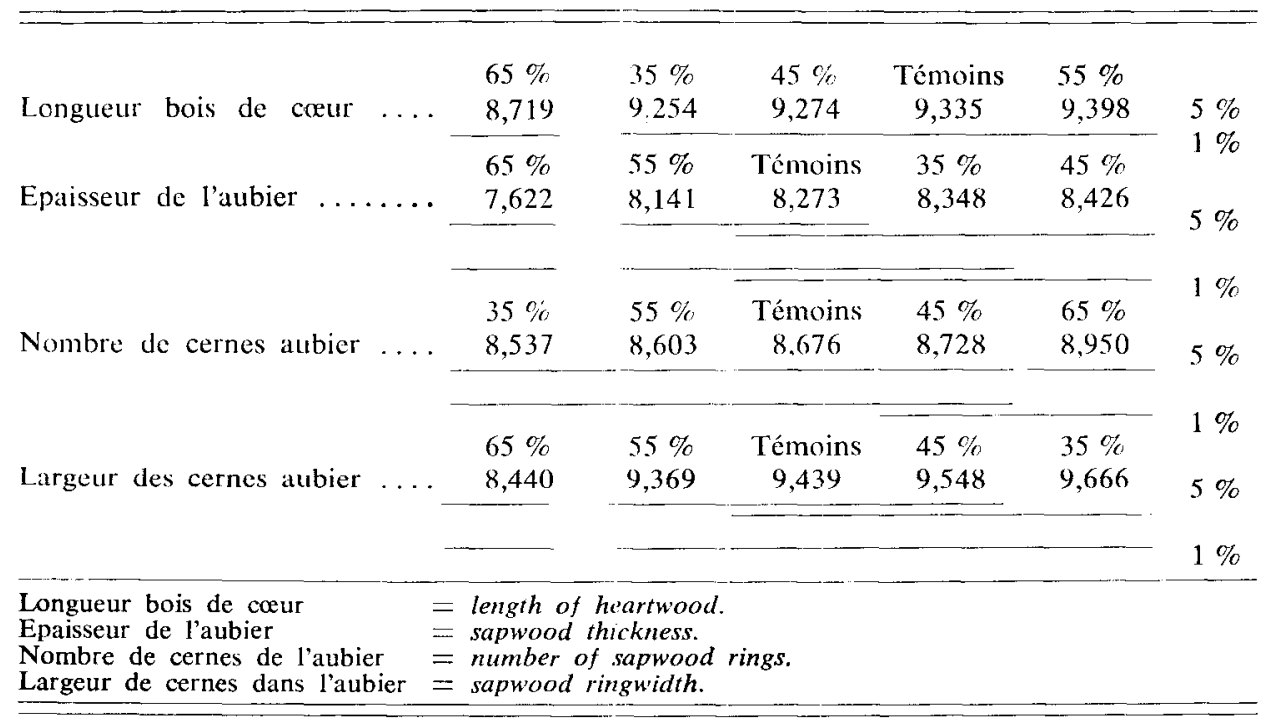

Une première remarque s'impose : les traitements ne sont jamais ordonnés suivant l'intensité des taux d'élagage, mais, en revanche, les arbres les plus sévèrement traités donnent toujours les valeurs les plus faibles ou les plus fortes, et se différencient toujours significativement des autres, au moins au seuil de signification de 5 p. 100 ; la raison est que, le dispositif vieillissant, beaucoup de branches qui avaient été laissées vivantes lors des opérations d'élagage ont dépéri, en sorte que le volume de houppier effectivement fonctionnel est maintenant à peu près le même pour toutes les catégories allant des témoins aux 55 p. 100, et que seuls les 65 p. 100 ont une surface assimilatrice plus réduite. Aussi bien le bois de cœur que l'aubier y sont moins étendus, ce qui explique que le pourcentage de la section du tronc occupé par le premier ne diffère pas significativement de celui trouvé pour les autres traitements. 
Les arbres ayant subi l'élagage le plus sévère ont un aubier formé de cernes plus étroits, mais plus nombreux; ce phénomène de quasi-compensation va bien dans le sens de la théorie de BamBEr déjà évoquée, de même que l'épaisseur globale réduite de ce même aubier, puisque la diminution du volume du houppier entraîne des besoins moindres de circulation de sève.

\subsection{Analyse de variance pour l'ensemble des caractéristiques} mesurées sur les prélèvements effectués à la hauteur de $1,30 \mathrm{~m}$

Le niveau inférieur est le seul où une analyse portant sur l'effet «traitement » peut être effectuée pour toutes les variables, y compris les diamètres longitudinaux et tangentiels des carottes de sondage à l'état frais : au niveau $6,50 \mathrm{~m}$ en effet, les arbres élagués à 65 p. 100 , dont l'analyse précédente a montré qu'ils étaient les seuls à se séparer nettement des autres, ont, du fait de leur diamètre plus faible, fait surtout l'objet de prélèvements diamétraux ( 3 individus seulement ont été sondés en deux fois en introduisant la tarière successivement côté Ouest et côté Est, comme cela a été fait systématiquement à $1,30 \mathrm{~m}$ ) ; l'analyse n'aurait donc pu être faite dans des conditions d'orthogonalité satisfaisante qu'en éliminant le traitement le plus intéressant.

Les résultats obtenus au seul niveau retenu sont résumés dans le tableau suivant :

TABLEAU 5

\begin{tabular}{|c|c|c|c|c|}
\hline \multicolumn{2}{|c|}{ Caractéristique analysée } & $\begin{array}{c}\text { Effet } \\
\text { traitement }\end{array}$ & $\begin{array}{l}\text { Effet } \\
\text { côté }\end{array}$ & Interaction \\
\hline \multicolumn{2}{|c|}{ Diamètre tangentiel frais $\ldots \ldots \ldots \ldots \ldots \ldots$} & N.S. & $50,14 * *$ & N.S. \\
\hline \multicolumn{2}{|c|}{ Diamètre longitudinal frais $\ldots \ldots \ldots \ldots \ldots$} & N.S. & $6,76 * *$ & N.S. \\
\hline \multicolumn{2}{|c|}{ Longueur radiale du bois de caeur $\ldots \ldots \ldots \ldots$} & $4,35 * *$ & $5,84^{*}$ & N.S. \\
\hline \multicolumn{2}{|c|}{ Epaisseur de laubier $\ldots \ldots \ldots \ldots \ldots \ldots \ldots$} & $13,86 * *$ & $11,00^{* *}$ & N.S. \\
\hline \multicolumn{2}{|c|}{ Nombre de cernes de l'aubier $\ldots \ldots \ldots \ldots \ldots \ldots$} & $4,11 * *$ & N.S. & N.S. \\
\hline \multicolumn{2}{|c|}{ Largeur des cernes dans l'aubier $\ldots \ldots \ldots \ldots \ldots$} & $18,20 * *$ & $6,43^{*}$ & N.S. \\
\hline \multicolumn{2}{|c|}{ Pourcentage de bois de cour $\ldots \ldots \ldots \ldots \ldots \ldots$} & N.S. & $12,59 * *$ & N.S. \\
\hline \multicolumn{2}{|c|}{ Retrait tangentiel $\ldots \ldots \ldots \ldots \ldots \ldots \ldots$} & N.S. & $10,45 * *$ & N.S. \\
\hline \multicolumn{2}{|c|}{ Retrait longitudinal $\ldots \ldots \ldots \ldots \ldots \ldots \ldots$} & N.S. & N.S. & N.S. \\
\hline \multicolumn{2}{|l|}{ Retrait radial ... } & N.S. & N.S. & $2,75^{*}$ \\
\hline \multicolumn{2}{|l|}{ Infra-densité . } & N.S. & $6,67 * *$ & N.S. \\
\hline \\
\hline \multicolumn{5}{|c|}{$\begin{array}{ll}\text { Caractéristique analysée } & =\text { property analyzed. } \\
\text { Diamètre tangentiel frais } & =\text { wet tangential diameter. }\end{array}$} \\
\hline \multicolumn{5}{|c|}{ Diamètre longitudinal frais $\quad=$ wet diameter in the grain direction. } \\
\hline \multicolumn{5}{|c|}{ Longueur radiale du bois de cour = radial length of heartwood. } \\
\hline \multicolumn{5}{|c|}{ Epaisseur de l'aubier $=$ sapwood thickness. } \\
\hline \multirow{2}{*}{\multicolumn{5}{|c|}{$\begin{array}{l}\text { Nombre de cernes de l'aubier } \\
\text { Largeur de cernes dans l'aubier }\end{array}$}} \\
\hline & $=$ sapwood ringwid & & & \\
\hline \multirow{2}{*}{\multicolumn{5}{|c|}{ Pourcentage de bois de coeur }} \\
\hline & $=$ tangential shrink & age. & & \\
\hline \multicolumn{5}{|c|}{$=$ longitudinal shrinkage. } \\
\hline \multicolumn{5}{|c|}{$=$ radial shrinkage. } \\
\hline \multicolumn{5}{|l|}{ Infra-densité } \\
\hline \multicolumn{5}{|l|}{ Effet traitement } \\
\hline \multicolumn{5}{|c|}{$=$ influence of the side. } \\
\hline Interaction & $=$ interaction. & & & \\
\hline
\end{tabular}


On ne trouve aucun effet «traitement» sur les diamètres à l'état frais, et notamment sur le diamètre tangentiel, dont on a prouvé qu'il est un excellent révélateur du bois de tension chez le peuplier.

Les 4 effets «traitement » significatifs observés sont les mêmes que ceux mis en évidence dans l'analyse précédente, et n'appellent pas de commentaire supplémentaire.

Il n'apparaît pas au niveau global d'influence du taux d'élagage sur le pourcentage de bois de cour (la valeur du « $F$ » correspondant est de 1,97, proche du seuil de signification de 5 p. 100); un test de comparaison de moyennes fait cependant apparaître des différences entre traitements significatives au seuil de 5 p. 100 , avec les résultats suivants :

\begin{tabular}{llllllll}
\hline \hline & $55 \%$ & $65 \%$ & Témoins & $35 \%$ & $45 \%$ \\
\hline$\%$ de bois de cour $\ldots \ldots \ldots$ & 32,10 & 32,03 & 30,91 & 30,89 & 30,29 \\
\hline
\end{tabular}

Aux deux plus forts taux d'élagage correspondent les pourcentages de bois de cœur les plus élevés, et une tendance se dégage donc à un effet positif du traitement sur la duraminisation, en conformité avec les données de la littérature précédemment citées.

Les deux diamètres des carottes à l'état frais sont soumis à un très fort effet «côté »; les valeurs obtenues sont les suivantes :

\begin{tabular}{l|c|c}
\hline & Côté Ouest & Côté Est \\
\hline Diamètre tangentiel $\ldots \ldots \ldots \ldots \ldots \ldots \ldots \ldots$ & $5084,1 \mu$ & $5106,0 \mu$ \\
Diamètre longitudinal $\ldots \ldots \ldots \ldots \ldots \ldots$ & $5202,9 \mu$ & $5204,7 \mu$ \\
\hline
\end{tabular}

On voit que les deux diamètres, et surtout le diamètre tangentiel sont plus faibles du côté Ouest, où le bois se trouve tendu sous l'effet des vents dominants, ce qui confirme, une fois de plus, la liaison négative entre bois de tension et diamètres des carottes à l'état frais.

Les autres effets «côté » significatifs concernent 5 variables pour lesquelles ils apparaissaient déjà dans l'analyse de variance à 3 niveaux du paragraphe précédent, avec des résultats tout à fait comparables (longueur du bois de coeur, pourcentage du bois de cour, infradensité et retrait tangentiel plus élevés et épaisseur de l'aubier plus faible du côté Ouest que du côté Est des tiges).

Une seule caractéristique nouvelle apparaît soumise à une influence de l'orientement dans la partie inférieure de la tige : la largeur des cernes de l'aubier qui passe de $8,859 \mathrm{~mm}$ à l'Ouest à $9,135 \mathrm{~mm}$ à l'Est, ce qui confirme que, contrairement au bois de compression, le bois de tension n'a pas toujours des accroissements annuels plus larges que le bois opposé des mêmes arbres. 


\subsection{Analyse des effets entrée et sortie de la tarière sur les diamètres des carottes de sondage}

Les sondages à $6,50 \mathrm{~m}$ ont été effectués, on l'a vu, de façon équilibrée (même nombre, à une unité près, de prélèvements avec entrée Est et avec entrée Ouest), en pensant qu'une même tarière, pénétrant dans une même zone de bois proche de l'écorce, pouvait donner des carottes de diamètre différent suivant que l'on se trouve du côté entrée ou du côté sortie de l'instrument dans le tronc. Il est intéressant de le vérifier, à la fois pour voir si les précautions prises dans la réalisation des sondages et dans l'interprétation des résultats étaient, a posteriori, justifiées, et, si cet effet supposé se trouve confirmé, pour en tenir compte dans les expériences futures.

On a donc procédé à des comparaisons de moyennes appariées entre les diamètres tangentiels et longitudinaux des carottes prélevées à $6,50 \mathrm{~m}$ dans les différents cas de figure qui se présentaient; leurs résultats sont donnés ci-dessous (diamètres exprimés en microns) :

TABLEAu 6

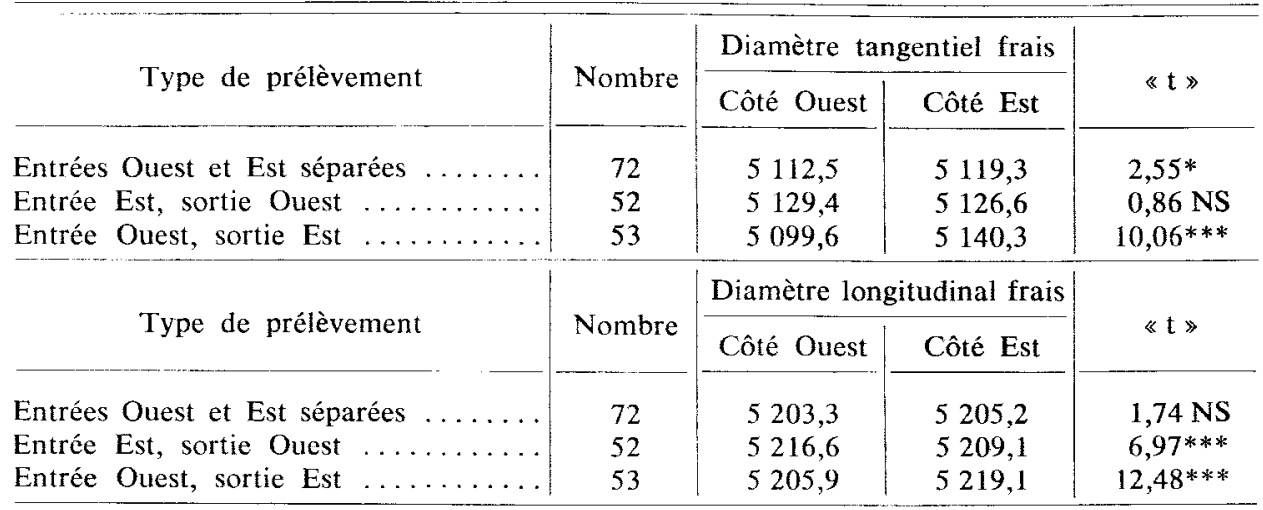

Type de prélèvement = kind of boring.

Entrées Ouest et Est séparées = separate west and east introductions.

Entrée Est, sortie Ouest = east introduction, west way out.

Entrée Ouest, sortie Est = west introduction, east way out.

Nombre $\quad$ number.

Diamètre tangentiel frais = wet tangential diameter.

Diamètre longitudinal frais $=$ wet diameter in the grain direction.

Côté Ouest = west side.

Côté Est = east side.

Concernant le diamètre tangentiel, on observe tout d'abord que, dans le cas de prélèvements séparés, le diamètre Ouest est significativement inférieur au diamètre Est, mais la différence est moindre qu'à $1,30 \mathrm{~m}(6,8$ microns contre 21,9$)$; ceci montre que le bois de tension se retrouve sur toute la longueur de la bille de pied du côté soumis aux vents dominants, mais que son importance diminue avec la hauteur. L'échauffement supposé de la tarière pendant son trajet dans l'arbre a pour 
effet d'entraîner une augmentation du diamètre de la carotte du côté sortie, en sorte que l'effet précédent se trouve complètement masqué quand la sortie se fait à l'Ouest, et, au contraire, considérablement amplifié lorsque l'on procède à un sondage diamétral de direction opposée.

Le phénomène est un peu différent, mais encore très net pour les diamètres dans le sens du fil du bois : il n'y a plus de différences significatives dans le cas de prélèvements séparés, mais, en revanche, l'augmentation du diamètre due à la situation de la zone de mesure côté sortie de la carotte est manifeste, puisque l'on trouve des différences de signe contraire, toutes deux significatives au seuil de 1 pour 1000 , suivant que la tarière a été introduite à l'Est ou à l'Ouest.

L'ensemble de ces résultats amène à déconseiller la pratique de sondages diamétraux dans toute expérience où les variables étudiées sont les diamètres, mesurés au micron près, des carottes de sondage, en raison du rôle prépondérant, vis-à-vis de toute autre source de variation, joué par l'emplacement de la zone de mesure par rapport au côté d'introduction de la tarière dans le déterminisme de ces mêmes diamètres.

Cet effet ne risque pas de se produire dans les sondages avec entrées Ouest et Est séparées, et la comparaison que l'on peut faire entre les carottes prélevées à $1,30 \mathrm{~m}$ et $6,50 \mathrm{~m}$ suivant cette modalité demeure donc possible ; elle montre que les diamètres tangentiels, aussi bien du côté tendu que du côté opposé, sont significativement plus forts au niveau supérieur, ce qui met en évidence une diminution du niveau des contraintes avec la hauteur.

\section{Commentaires et conclusion}

Les observations consignées ci-dessus font apparaître une influence faible ou nulle de l'élagage sur la formation de bois de tension d'une part, et sur la duraminisation d'autre part, en opposition avec les données de la littérature rappelées en introduction.

La méthodologie utilisée ne devrait pas être mise en cause pour expliquer ces résultats : pour l'estimation du bois de coeur, la prise en compte de deux rayons opposés suivant un orientement correspondant à la plus grande anisotropie devrait fournir une précision d'estimation au moins aussi bonne que celle que l'on peut obtenir sur un plus grand nombre de directions ou par mesure directe de surfaces sur un petit nombre d'arbres; quant à l'appréciation indirecte du bois de tension à partir des diamètres des carottes de sondage à l'état frais, qui reposait déjà sur plusieurs expériences aux résultats parfaitements concordants, elle se trouve confortée ici par les différences relevées entre côté tendu et côté opposé.

Au reste, si l'on s'en tient aux résultats de SACHSSE (ceux de SACRE étant difficilement interprétables en raison de conditions trop différentes de milieu et de compétition entre arbres élagués et témoins), la contradiction n'est pas aussi nette qu'il n'apparaît à première vue :

- en ce qui concerne la duraminisation, l'effet de l'élagage n'est significatif que si l'on prend en considération à la fois les trois hybrides entre peupliers noirs et peupliers baumiers, et les 2 hybrides euraméricains ; si l'on se limite à ces derniers (auxquels se rattache le peuplier I 214), les différences relevées sont très faibles et 
voisines de celles trouvées ici; même en valeur absolue, les pourcentages trouvés sur Regenerata (33,3 p. 100 pour les arbres élagués et 30,6 p. 100 pour les témoins) sont parfaitement comparables à ceux obtenus en Forêt de Retz sur 1214 (32,1 p. 100 en moyenne pour les élagués à 55 p. 100 et 65 p. 100 contre 30,7 p. 100 pour les témoins et les élagués à 35 p. 100 et 45 p. 100);

- la situation est à peu près la même en ce qui concerne le bois de tension : si l'on s'en tient aux peupliers euraméricains, il n'y a, d'après les données détaillées publices par SACHSSE (op. cit.), aucune différence significative entre élagués et témoins, et on n'en obtient une que si l'on fait intervenir les hybrides peupliers noirs $\times$ peupliers baumiers, et, en particulier, le cultivar Wettstein 264 .

On pourrait certes objecter à l'expérience de Retz que l'étude a été entreprise à un moment où, du fait du dépérissement des branches inférieures sur les arbres les moins élagués, les divers traitements ne se différencient plus guère les uns des autres du point de vue de leur masse foliaire ou de leur croissance; cependant, le traitement à 65 p. 100 qui se distingue bien des autres n'apparaît nullement comme ayant davantage de bois de tension qu'eux. D'autre part, l'essentiel est ce qui se passe dans la pratique populicole courante; la séquence d'interventions suivie en Forêt de Retz, élagages à 7,12 et 15 ans, est, de ce point de vue, assez réaliste, même si des interventions plus précoces sont parfois pratiquées (Bonduelle, 1971), et l'étude du bois à 21 ans également, puisque cela correspond à un âge où on peut envisager une récolte en bois d'œuvre. Il vient automatiquement un moment où on arrête les opérations d'élagage qui deviennent trop onéreuses en raison du niveau élevé auquel il faudrait intervenir; il est normal que, la croissance en hauteur continuant, les branches les plus basses meurent ; cela se produit naturellement dans les peupleraies, même cultivées intensivement, et seul importe donc de savoir si, en l'occurrence, on trouve plus de bois de tension, lors de l'exploitation, sur les arbres ayant été antérieurement élagués ou non. Pour le I 214, dans les conditions de la Forêt de Retz, la réponse est négative.

Bien que l'on ne dispose pas de renseignements précis, il semble que dans l'expérience de SACHSSE, les arbres aient été élagués annuellement à partir de 5 ans, et que les études du bois formé aient été faites à 9 ans; il n'est pas impossible que, sur des sujets aussi jeunes, il y ait effectivement plus de bois de tension sur les individus élagués, mais, si le phénomène s'estompe avec l'âge, il ne peut être considéré comme très grave, puisque la partie centrale des troncs ne présente qu'un intérêt industriel limité.

Quoi qu'il en soit, il est prudent de ne pas extrapoler les résultats consignés ici à ses cultivars autres que le I 214, puisqu'il apparaît, au vu de l'expérience de SACHSSE que les effets de l'élagage sur la formation de bois de tension peuvent varier d'un clone à l'autre, et puisque, par ailleurs, une étude, non encore publiée, de la Station de Recherches sur la Qualité des Bois et de l'Association Forêt-Cellulose (AFOCEL) met en évidence une forte héritabilité au sens large de l'aptitude des peupliers à produire du bois de tension. Il serait donc intéressant, lorsque le matériel expérimental nécessaire sera disponible, d'analyser à nouveau, sur un échantillonnage suffisant, les conséquences de l'ablation de branches vivantes sur l'élaboration du bois de tension, en se focalisant sur des clones de la Section Tacamahaca qui paraissent avoir une propension toute particulière à en produire.

Reçu le 20 mars 1984.

Accepté le 15 juin 1984. 


\section{Remerciements}

MM. Jean-René Perrin, Jean Perrin, Claude Houssement ont procédé aux prélèvements de terrain, et $\mathbf{M}^{\text {me }}$ Danièle AUBERT a mené à bien l'ensemble des calculs statistiques nécessaires; que tous soient vivement remerciés ici.

\section{Summary}

Influence of pruning on the heartwood formation, the production of tension wood and some other wood properties of 1214 poplar

Four increments cores have been taken (at 1.30 and $6.50 \mathrm{~m}$ above ground, both on the western and the eastern sides of the boles) in 177 I 214 poplars pruned at different intensities up to $65 \mathrm{p}$. 100 , to know the effects of the pruning treatment, more particularly on the occurence of tension wood (indirectely estimated from the tangential diameter of the increment cores in wet condition) and on the heartwood formation. The main results are as follows :

- Heartwood is thinner and sapwood larger, tangential shrinkage and specific gravity higher on the western side of the trees, $i$. e. that of the prevailing winds;

- At $6.50 \mathrm{~m}$, sapwood rings are fewer, but they are wider than at breast height ;

- Pruning only influences the different parameters related to heartwood formation : in the 65 p. 100 treatment, sapwood lies on more numerous, but narrower rings;

- There is a tendency for the severely pruned trees $(55$ p. 100 and 65 p. 100) to have a higher percentage of heartwood;

- The amount of tension wood is higher on the western side in relation with the tension stresses due to the wind, but is quite independent of the pruning treatment.

\section{Références bibliographiques}

Bamber R.K., 1975. Comparative studies or sapwood and heartwood. Thèse Doctor of Philosophy, Macquarie University, February.

Bonduelle P., 1971. Etude théorique et pratique de l'élagage du peuplier I 214. Rapport annuel de l'A.F.O.C.E.L., 293-345.

Ferrand J.Ch., 1981. Recherches des solutions pratiques à apporter aux problèmes posés par les contraintes de croissance des arbres forestiers. Thèse de Docteur-Ingénieur en Sciences du Bois, I.N.P.L., Nancy, 11 décembre.

Keller R., 1979. Premiers résultats d'une expérience d'élagage du peuplier I 214. Ann. Sci. for., 36 (1), 59-92.

OKuYama T., 1983. Report on some experimental works on growth stress in wood. Mesure locale des contraintes de croissance près du cambium. Centre Technique du bois, Action de Recherche, $\mathrm{H}^{\circ} 208$, Paris.

Ollinmai P.J., 1956. Acta for. fenn., 64 (3).

Perrin J.R., Ferrand J.Ch., 1984. Automatisation des mesures sur carottes de sondage de la densité du bois, de son retrait et des contraintes de croissance. Ann. Sci. forest., 41 (1), 69-86.

Polge H., Thiercelin F., 1979. Growth stress appraisal through increment core measurements. Wood Science, 12 (2), 86-92.

Pourtet J., 1961. La culture du peuplier. J.B. Baillière et Fils, Paris, $2^{c}$ édition.

SACHSSE H., 1965. Untersuchungen über den Einfluss der Astung auf die Farbkern- und Zugholzausbildung einiger Pappelsorten. Holz als Roh- und Werkstoff, 23 (11), 425-434.

SaCre E., 1959. Le bois de tension des peupliers. Bull. Inst. Agron. Gembloux, 27 (4), $435-444$.

SaCre E., 1974. Proportion de faux cour et de bois de tension dans les peupliers « Robusta » élagués et non élagués. Document Station de Technologie Forestière, Gembloux.

Trenard Y., Gueneau P., 1975. Relations entre contraintes de croissance longitudinales et bois de tension dans le hêtre. Holzforichung, 29 (6), 217-223. 